\title{
A static analogy for 2D tolerance analysis
}

\author{
Antonio Armillotta \\ Politecnico di Milano, Dipartimento di Meccanica \\ antonio.armillotta@polimi.it
}

\begin{abstract}
Purpose - This paper presents a method for the tolerance analysis of mechanical assemblies that is suitable to nonlinear problems where explicit functional equations are difficult or even impossible to write down. Such cases are usually modelled by linearised tolerance chains, whose coefficients (or sensitivities) are calculated from assembly data.

Design/methodology/approach - The method is based on the free-body diagrams of force analysis, which are shown to be related to the sensitivities of linearised functional equations. Such an analogy allows the conversion of a tolerance chain into a corresponding static problem, which can be solved by common algebraic or graphical procedures.
\end{abstract}

Findings - The static analogy leads to a correct treatment of tolerance chains, as the analysis of several examples has confirmed by comparison to alternative methods.

Research limitations/implications - Currently, the method has only been tested on two-dimensional chains of linear dimensions for assemblies with nonredundant kinematic constraints among parts.

Practical implications - The proposed method lends itself to ready application by using simple operations with minimal software assistance. This could make it complementary to current methods for calculating sensitivities, which are mathematically complex and require software implementation for deployment in industrial practice.

Originality/value - Analogy with force analysis, which has not been previously highlighted in the literature, is a potentially interesting concept that could be extended to a wider range of tolerancing problems.

\section{Keywords}

Tolerance analysis, tolerance chain, stackup coefficients, force analysis, free-body diagram

\section{Introduction}

Tolerance analysis is an important task in the design of mechanical assemblies and has received considerable attention in the literature (Shen et al., 2005; Shah et al., 2007; Polini, 2009). Tolerance analysis generally consists of studying one or more tolerance chains, each of which associates a functional requirement on the assembly with a set of tolerances specified on individual parts; the mathematical model of a tolerance chain helps to detect situations where manufacturing errors allowed by part tolerances are likely to violate the required assembly tolerance. From this basic objective, however, the problem has been defined differently according to the needs of specific applications. Differences can involve properties of parts and assemblies (the geometry of the features 
and relations), types of tolerance specifications (dimensional or geometric) and assumptions on tolerance stackup (worst-case or statistical). As a consequence, several methods have been proposed for solving tolerance analysis problems under different sets of assumptions.

At the lowest level of complexity, the analysis includes only tolerances on part dimensions without considering deviations in geometric characteristics. At the expense of losing consistency with the principles of GD\&T (Geometric Dimensioning and Tolerancing), tolerance chains are easier to model with explicit functional equations. One-dimensional tolerance chains, which apply to most cases encountered in design practice, involve linear relationships among dimensions and requirements and are easily solved by well-known charting procedures (Fischer, 2004). For two- or three-dimensional problems, however, tolerance chains are often nonlinear, and functional equations cannot be written explicitly for assemblies of moderate complexity. In these cases, a common approach consists of linearising the functional equations and calculating the sensitivities (i.e., derivatives of requirements with respect to dimensions) through algorithms based on geometric reasoning, which include the vector loop method (Chase et al., 1994; Chase et al., 1997; Gao et al., 1998) and methods based on small displacement torsors (Bourdet et al., 1995; Bourdet and Ballot, 1995; Ballot and Bourdet, 1997; Legoff et al., 1999). As it is impractical for a designer to perform the required calculations by hand, analytical procedures for evaluating sensitivities are usually implemented using software tools, which automate the calculations through direct $\mathrm{CAD}$ integration or from interactively built abstract geometric models (Prisco and Giorleo, 2002; Shen, 2003; Chiesi and Governi, 2003).

When the analysis includes geometric tolerances, the above approach must be adapted to the diversity of the geometric characteristics of interest for mechanical assemblies. For a subset of tolerance types defined in the GD\&T standards, such an extension is feasible for charting procedures and procedural linearisation methods, as proven in several papers (Chase et al., 1994; Giordano and Duret, 1993; Teissandier et al., 1999). Further approaches have been proposed for a comprehensive treatment of geometric deviations, including the transform chain method (Mantripragada and Whitney, 1998; Mantripragada and Whitney, 1999), the Jacobian-torsor method (Desrochers and Rivière, 1997; Desrochers et al., 2003), the method based on the representation model of Technologically and Topologically Related Surfaces (TTRS; Salomons et al., 1996), the method of virtual joints (Lafond and Laperrière, 1999), the variational method (Gupta and Turner, 1993; Sodhi and Turner, 1994) and the T-maps model (Shen et al., 2005; Shah et al., 2007). Relying upon software tools is again necessary and results in even more difficulties related to availability and learning.

This work aims to discover shortcuts to solving nonlinear tolerance chains using simple calculations with minimal software assistance, which would be straightforward for most mechanical designers. The attempt may be beneficial for two-dimensional tolerance chains, which often cannot be solved through charting or explicit functional equations. The results presented in the paper show that such shortcuts exist due to a property of sensitivities that allows their calculation by a procedure similar to graphical force analysis. 
The attempt to find analogies with other mechanical analysis problems is not new. In previous work from the team that developed the vector loop method (Huo, 1995; Faerber, 1999), an analogy with kinematic analysis was shown to allow the calculation of sensitivities from the relative velocities of the links in a mechanism. Although these studies investigate a more specific and complex variation of the tolerance analysis problem (sensitivities are time dependent due to the degrees of freedom of the mechanism), they also aim to exploit known graphical and numerical procedures as an alternative to computer-aided tolerancing tools.

As will be discussed below, the proposed approach can only be applied in special cases involving three basic assumptions: a) dimensional tolerances, b) linear dimensions and functional requirements, and c) no kinematic redundancy in assembly relations. This paper will describe the analysis procedure and demonstrate its validity on several examples to show that such limitations may be acceptable for practical applications.

\section{Assumptions and definitions}

A two-dimensional tolerance chain is defined on an assembly whose properties can be described to be in an $x-y$ plane for the purpose of analysis. This situation occurs in many cases where a representative section of the assembly drawing carries all the necessary information concerning the shape and dimensions of the parts as well as the contact relations between the parts. Although there are few limits to the diversity of the geometric configurations of an assembly, a basic restriction will be assumed for the functional dimensions and functional requirements: they will only be expressed as linear distances; the angular dimensions are excluded. The need to satisfy such a condition could require a preliminary revision of the part drawings to replace angular dimensions with linear dimensions at selected points (e.g., the extreme points of a straight line edge); a similar point-based simplification could also be made for the functional requirements defined by angular assembly dimensions. Additionally, contact relations will be assumed to be of any possible type that can occur among common planar primitives (mainly straight line segments and arcs of circles) including their tangency and intersection points.

A tolerance chain is a representation of how a functional requirement $x$ depends on a known set of functional dimensions $d_{i}(i=1, \ldots n)$. Due to manufacturing errors in parts, each dimension has a deviation from its nominal value:

$\Delta d_{i}=d_{i}-\bar{d}_{i} \quad(i=1, \ldots n)$

As a consequence, a deviation will occur on the functional requirement:

$\Delta x=x-\bar{x}$

In the general case defined by the above assumptions, evaluating $\Delta x$ is difficult because the functional equation 


$$
x=f\left(d_{1}, \ldots d_{n}\right)
$$

is nonlinear and possibly unknown. However, the equation can be linearised by a first-order Taylor approximation because the deviations on the dimensions are small compared to the nominal values:

$$
\begin{aligned}
& \bar{x}=\sum_{i=1}^{n} s_{i} \bar{d}_{i} \\
& \Delta x=\sum_{i=1}^{n} s_{i} \Delta d_{i}
\end{aligned}
$$

Linearisation is feasible if one can evaluate the sensitivities of $x$ with respect to the dimensions $d_{i}$ :

$$
s_{i}=\left.\frac{\partial x}{\partial d_{i}}\right|_{\bar{d}_{1}, \ldots, \bar{d}_{n}}(i=1, \ldots n)
$$

In a tolerance analysis problem, the sensitivities allow for the evaluation of the resulting tolerance $T$ on the requirement from the tolerances $T_{i}$ assigned to the dimensions. Depending on the applications, this evaluation is often performed using either the worst-case (linear) or statistical (root-sum-square, RSS) stackup:

$$
\begin{aligned}
& \text { linear: } T=\sum_{i=1}^{n}\left|s_{i}\right| T_{i} \\
& \text { RSS: } T=\sqrt{\sum_{i=1}^{n} s_{i}^{2} T_{i}^{2}}
\end{aligned}
$$

The problem investigated in this paper is how the sensitivities can be evaluated without explicit knowledge of the functional equation, using only reasoning on the geometric information that can be extracted from a planar geometric description of the assembly.

\section{Proposed procedure}

A clue to the evaluation of the sensitivities can be sought from well-known properties of equilibrium conditions on rigid bodies. Figure 1 shows a planar body whose degrees of freedom are constrained by three frictionless point contacts with adjacent bodies. Under an external force $F$ applied to the boundary of the body, the three constraints react with the forces $F_{1}, F_{2}$, and $F_{3}$. Due to the assumption of a rigid body and thus neglecting any force-induced deformations, the small displacements $\delta_{1}, \delta_{2}$, and $\delta_{3}$ of the contact points along the directions of the reaction forces cause a displacement $\delta$ of the point of force application, which is assumed to be positive if it is opposite to $F$. In accordance with the principle of virtual work for rigid bodies (e.g., Timoshenko and Young, 1983), the total work of the four forces equals zero because the body does not change its potential or kinetic energy, yielding the following relationship among the four displacements: 
$\delta=\frac{F_{1}}{F} \delta_{1}+\frac{F_{2}}{F} \delta_{2}+\frac{F_{3}}{F} \delta_{3}$

Therefore, displacements along directions of constraints contribute to a displacement along the direction of an external force in proportion to the corresponding reaction forces. This suggests a possible analogy with tolerance analysis. If the displacements $\delta_{i}$ are now considered to be manufacturing errors along the directions associated with the functional dimensions of a part, the resulting displacement $\delta$ can be viewed as the stackup of these errors given the contact relations with other parts. The sensitivities of $\delta$ with respect to the $\delta_{i}$ can thus be evaluated as the reaction forces of adjacent parts under a unit external force applied to the part at the same point and along the same direction as that defined for the error. If the directions associated with the contact points are not parallel to the functional dimensions, one can still suppose that the sensitivities can be evaluated as components of the reaction forces along the directions of the dimensions.

Considering all the parts of an assembly, the propagation of small displacements through the contact points can be viewed in analogy to the transmission of reaction forces among parts in static equilibrium. The sensitivities of an error with respect to all functional dimensions defined for the assembly can thus be evaluated by analysing the equilibrium conditions on free-body diagrams created for individual parts. Each functional requirement will be generally associated with two unit external forces with the same line of action and opposite orientations, which are acting at reference points on the two parts that are directly involved in the requirement.

The above considerations lead to the following procedure for the evaluation of sensitivities in a linearised two-dimensional tolerance chain, as outlined in Figure 2 (for the sake of simplicity, a trivial one-dimensional case is used):

a) The requirement to be analysed is defined as a linear dimension along a certain direction, measured between reference points of two different parts (end parts).

b) Two opposite unit forces are applied to the end parts at the same points and along the same direction of the requirement. Each force is oriented consistently with the corresponding arrow of the dimension that represents the requirement on the assembly drawing, although a different convention would have no influence on the subsequent calculations.

c) The equilibrium conditions under the imposed forces are analysed by constructing free-body diagrams for all parts of the assembly. Each diagram includes the lines of action of the reaction forces associated with the contact relations, which are assumed to be frictionless and bilateral (i.e., with no loss of contact allowed between parts).

d) Once reaction forces are evaluated for each part, they are decomposed along the directions of the functional dimensions. This operation yields the sensitivities of the requirement with respect to the same dimensions. The sign of each sensitivity depends on the orientations of the 
corresponding force components, which act along the functional dimensions as axial loads act on one-dimensional structural members (positive tension and negative compression).

The procedure is likely to sound familiar to mechanical designers, who are accustomed to using freebody diagrams in the force analysis of planar structures and mechanisms. If the parts are underconstrained or exactly constrained, the reaction forces are easily calculated by writing translational and rotational equilibrium equations. In most cases, however, the free-body diagrams can be analysed by the graphical decomposition of forces, possibly with the aid of a 2D drafting tool so that the force intensities could be queried with better precision. This task is relatively simple even when the assembly is too complicated for an analytical calculation of the sensitivities. The limitation of nonredundant contact relations is common to all tolerance analysis methods that hold the rigidbody assumption and do not account for part compliance.

\section{Validation}

A few conceptual examples are given below to demonstrate the proposed procedure and to verify its correctness through comparison with alternative methods.

\subsection{A basic example with explicit functional equation}

Figure 3a illustrates a simple two-dimensional tolerance chain, which has already been used as a test case in several papers on tolerance analysis (Marziale and Polini, 2009; Marziale and Polini, 2010). Two circular parts are in contact with each other and rest against the edges of a rectangular box. The requirement of interest is the distance $x$ between the upper circle and the top edge of the rectangle. The selected dimensions are the radii $r_{1}$ and $r_{2}$ of circles (the more appropriate choice of diameters will be discussed later) and the lengths $h$ and $b$ of the rectangle edges.

As shown in Figure 3b, the procedure begins by drawing an assembly diagram where two opposite unit forces $F$ act on the end parts related to requirement $x$; the dimension line of $x$ on the assembly drawing dictates the points, lines of action and orientations of the two forces. The free-body diagrams are then drawn for all parts to analyse their equilibrium conditions. As usual in force analysis, the inspection of all diagrams in proper order yields the reaction forces acting among the parts. Although such an operation is likely to be performed graphically in most cases, this assembly is simple enough for an analytical calculation of the force magnitudes as a function of $\alpha$ (an angle depending on the dimension values).

The final task of the procedure consists of decomposing the forces acting on individual parts along the directions of the functional dimensions, which, as previously mentioned, is performed in the same way as tensile and compressive internal forces are evaluated from free-body diagrams on beams, trusses and frames. Specifically, the rectangular box can be regarded as a frame that is subject to four equilibrating forces. Any of the two vertical members with length $h$ is pulled with a tensile force equal 
to $F$; according to the static analogy, such an internal force corresponds to the sensitivity of the functional requirements with respect to dimension $h$ :

$$
\frac{\partial x}{\partial h}=F=1
$$

Similarly, any of the two horizontal members with length $b$ bears a tensile force equal to $F \tan \alpha$, which allows calculation of the sensitivity of $x$ with respect to $b$ :

$$
\frac{\partial x}{\partial b}=F \tan \alpha=\frac{b-\left(r_{1}+r_{2}\right)}{\sqrt{2 b\left(r_{1}+r_{2}\right)-b^{2}}}
$$

The circular part with radius $r_{1}$ is subject to three radial, inward-directed forces. If each of the forces were equilibrated by a reaction at the centre of the circle, a compression of equal value would act along its direction; according to the static analogy, such an internal force should correspond to the sensitivity of $x$ with respect to the radius on that direction. As dimension $r_{1}$ is defined for all three directions, the sum of the three compressive forces yields the sensitivity of $x$ with respect to $r_{1}$ :

$$
\frac{\partial x}{\partial r_{1}}=-F-F \tan \alpha-\frac{F}{\cos \alpha}=-1-\frac{b}{\sqrt{2 b\left(r_{1}+r_{2}\right)-b^{2}}}
$$

The forces acting on the circular part with radius $r_{2}$ are equivalent to those of the previous part in both intensity and direction. The sensitivity of $x$ with respect to $r_{2}$ is thus equal to that calculated above:

$$
\frac{\partial x}{\partial r_{2}}=\frac{\partial x}{\partial r_{1}}=-1-\frac{b}{\sqrt{2 b\left(r_{1}+r_{2}\right)-b^{2}}}
$$

As a proof of correctness, the same values for the sensitivities are found by differentiation of the explicit functional equation, which is easily written for such a simple assembly:

$$
x=h-\left(r_{1}+r_{2}\right)-\sqrt{2 b\left(r_{1}+r_{2}\right)-b^{2}}
$$

\subsection{A more complex example with graphical verification}

The tolerance chain depicted in Figure $4 \mathrm{a}$ is similar to the previous example, but slightly more complicated, to the extent that an explicit functional equation would be much harder to write. Requirement $x$ is related to dimensions $h, b, r_{1}, r_{2}, r_{3}$, and $r_{4}$, whose nominal values are indicated on the assembly drawing. Again, unit forces $F$ are applied to the end parts to define an assembly force diagram (not shown). As shown on the free-body diagrams in Figure $4 \mathrm{~b}$, the equilibrium conditions of the five parts (four circles and a rectangle) are analysed graphically with the help of drafting software to obtain a precise decomposition of forces. This operation yields the reaction forces $F_{1}, F_{2}, \ldots F_{8}$, which, as before, are then projected along the directions of the functional dimensions. The following values for the sensitivities are found: 
$\mathbf{s}=\left[\begin{array}{l}\partial x / \partial h \\ \partial x / \partial b \\ \partial x / \partial r_{1} \\ \partial x / \partial r_{2} \\ \partial x / \partial r_{3} \\ \partial x / \partial r_{4}\end{array}\right]=\left[\begin{array}{c}F \\ F_{5} \\ -F-F_{1}-F_{2} \\ -F_{4}-F_{7}-F_{8} \\ -F_{2}-F_{3}-F_{4} \\ -F_{3}-F_{5}-F_{6}\end{array}\right]=\left[\begin{array}{c}1.00 \\ 0.75 \\ -2.86 \\ -0.63 \\ -2.53 \\ -2.54\end{array}\right]$

Lacking a functional equation, the correctness of these results is verified by graphical construction with the precision allowed by the drafting tool. The assembly is redrawn with some deviations with respect to the nominal values, as shown in Figure $4 \mathrm{c}$, thus yielding the following deviation on the requirement:

$\Delta x=x-\bar{x}=6.28-6.88=-0.60$

Such a value is in good accordance with the deviation resulting from the calculated sensitivities:

$\Delta x=\mathbf{s}^{\mathrm{T}} \cdot\left[\begin{array}{c}-0.1 \\ 0.2 \\ -0.1 \\ -0.1 \\ 0.1 \\ 0.1\end{array}\right]=-0.62$

A couple remarks on the calculation of the reaction forces can be added with reference to Figure $5 \mathrm{a}$, which shows the free-body diagram for one of the parts in the above example. As detailed in Figure $5 b$, the drafting tool (DraftSight ${ }^{\circledR}$ in this case) allows the force polygon for the decomposition of the external force $F$ along the directions associated to constraints 1 and 2 to be drawn interactively; after the graphical construction has been completed, the measuring capability of the tool provides the length of each vector and thus, in an appropriate scale, the intensity of each reaction force. As in all force analysis problems, the graphical construction could be replaced by an analytical method based on the direct solution of the equilibrium equations. A method derived from screw theory (Hunt, 1978) associates each constraint with a unit vector $\mathbf{v}_{i}$ along the inward-directed normal to the surface at the point of contact. In a planar case, the location and intensity of each unit vector is described by a threeelement vector (wrench) of the type

$$
\mathbf{w}_{i}=\left[\begin{array}{lll}
v_{x i} & v_{y i} & m_{i}
\end{array}\right]^{\mathrm{T}}
$$

where $v_{x i}$ and $v_{y i}$ are the components of $\mathbf{v}_{i}$ along the coordinate axes $x$ and $y$, and $m_{i}$ is the moment of $\mathbf{v}_{i}$ about the origin of the coordinate frame. If the wrenches are joined in a matrix

$$
\mathbf{W}=\left[\mathbf{w}_{i}\right]
$$

the equilibrium condition can be written as 


$$
\mathbf{W} \cdot \mathbf{f}=-\mathbf{w}_{\mathrm{F}}
$$

where $\mathbf{w}_{\mathrm{F}}$ is the wrench associated with the external force $F$, and the elements of vector $\mathbf{f}$ are the unknown intensities of the reaction forces. In the example (Figure $5 \mathrm{c}$ ), the above equation becomes

$$
\left[\begin{array}{cc}
1 & -\sin 33.34^{\circ} \\
0 & \cos 33.34^{\circ} \\
0 & 0
\end{array}\right] \cdot\left[\begin{array}{l}
F_{1} \\
F_{2}
\end{array}\right]=-\left[\begin{array}{c}
0 \\
-F \\
0
\end{array}\right]
$$

and yields the solution already found, $\left(F_{1}=0.658, F_{2}=1.197\right)$. The fact that screw theory has been widely used for the kinematic analysis of mechanisms (e.g., Ohwoworiole and Roth, 1981) suggests that the proposed approach is somehow related to existing methods for tolerance analysis, most of which exploit the mathematical formulations derived from that context.

\subsection{An example with a known solution}

For a comparison with the full-featured approaches to tolerance analysis, Figure 6a shows the 'geometric block' already used in the literature as an illustrative example for the vector loop method (Chase et al., 1994). Three parts with different shapes (a circle, a rectangle and a piecewise linear profile made by horizontal and vertical segments) have some clearly defined contact relations at their tangency or intersection points. The vertical distance $x$ between the centre of the circle and the lowest horizontal line segment is the functional requirement of interest, which is related to the functional dimensions $a, b, c, d, e$ and $f$, whose nominal values are indicated beside the assembly drawing.

As shown in Figure 6b, the requirement corresponds to an upward unit force $F$ acting at the centre of the circle and to its downward equilibrating force acting on the lowest horizontal line. As in the previous case, the free-body diagrams of the three parts are graphically inspected to evaluate the reaction forces $F_{1}, F_{2}, F_{3}$ and $F_{4}$. These are eventually decomposed along the directions of the functional dimensions, thus yielding the following values for the sensitivities:

$$
\left[\begin{array}{l}
\partial x / \partial a \\
\partial x / \partial b \\
\partial x / \partial c \\
\partial x / \partial d \\
\partial x / \partial e \\
\partial x / \partial f
\end{array}\right]=\left[\begin{array}{c}
F_{1}+F_{2} \\
F_{2} \\
F_{4 y} \\
F_{3 y} \\
-F_{4 x} \\
-F_{2}
\end{array}\right]=\left[\begin{array}{c}
1.31 \\
1.04 \\
0.26 \\
0.74 \\
-0.07 \\
-0.27
\end{array}\right]
$$

Considering the precision of graphical constructions, these results are in good accordance with those reported in (Chase et al., 1994), where the same sensitivities are calculated by the vector loop method: 


$$
\left[\begin{array}{l}
\partial x / \partial a \\
\partial x / \partial b \\
\partial x / \partial c \\
\partial x / \partial d \\
\partial x / \partial e \\
\partial x / \partial f
\end{array}\right]=\left[\begin{array}{c}
1.3098 \\
1.0367 \\
0.2581 \\
0.7419 \\
-0.0705 \\
-0.2731
\end{array}\right]
$$

\section{Applications}

As stated before, the use of a static analogy for tolerance analysis is subject to several limitations at the current state of development. Variables in tolerance chains are restricted to linear dimensions, whose directions suggest the correct calculation of the sensitivities as tensile/compressive forces. Deviations on angular dimensions and geometric characteristics are less easily related to contact forces and are thus excluded from the analysis. Overconstraining of parts is also excluded, thus reducing tolerance analysis to a purely geometrical problem.

Although the obvious need for completeness and compliance to modern GD\&T standards would require extension to remove such limitations, it is supposed that the procedure can find successful applications in mechanical design. Such a belief must be verified by proving that certain realistic configurations of assemblies can actually be treated within the application scope of the procedure. Further examples are given below to demonstrate this effort.

In the design of planar linkages, overconstraining is usually avoided and part connections (hinges, sliders, etc.) are easily related to reaction forces along the given directions. These conditions seem to match the assumptions of the procedure, as can be demonstrated for a typical tolerance analysis problem, the evaluation of clearances in hinged joints. Figure 7a shows two links connected to a pin, with a clearance fit between hole diameters $D_{1}$ and $D_{2}$ and pin diameter $d$. As clearly shown in Figure $7 \mathrm{~b}$, the maximum relative displacement of hole centres is related to the part dimensions by the following equation:

$$
x=\frac{D_{1}}{2}-d+\frac{D_{2}}{2}
$$

This equation is consistent with the calculation of sensitivities through the free-body diagrams for the three parts (Figure 7c). Opposite unit forces $F$ acting along the direction of $x$ induce reactions of equal intensities at contact points, which yield the following requirement sensitivities with respect to the three dimensions: 


$$
\begin{aligned}
& \frac{\partial x}{\partial D_{1}}=\frac{1}{2} \frac{\partial x}{\partial r_{1}}=\frac{F}{2}=0.5 \\
& \frac{\partial x}{\partial d}=-F=-1 \\
& \frac{\partial x}{\partial D_{2}}=\frac{1}{2} \frac{\partial x}{\partial r_{2}}=\frac{F}{2}=0.5
\end{aligned}
$$

Figure 8 illustrates the analysis of a planar linkage of realistic complexity. Here, the vertical distance $x$ from the top platform to the bottom frame is to be controlled with respect to eighteen functional dimensions selected on seven parts (platform, rod, actuator and four pins). Once opposite unit forces $F$ are suitably defined on end parts, free-body diagrams are created and inspected to find the reaction forces $F_{1}$ and $F_{2}$. These are then projected along the directions of functional dimensions to find the internal forces, which provide the values of sensitivities shown in Table 1. For different configurations of the linkage as a function of actuator length, new free-body diagrams can be readily created to calculate the effects on the sensitivities.

Although they do not include any geometric tolerance that might be specified on parts, the above results are sufficient for an RSS stackup analysis of a given set of tolerances on the functional dimensions. The procedure provides an easy way to evaluate the contributions of different types of dimensions (lengths of links, positions of joints, thicknesses, pin and hole diameters) to the overall variation of the functional requirement.

\section{Conclusions}

The use of computer-aided tools based on three-dimensional CAD modelling is increasingly recognised as essential for tolerance analysis on complex assemblies. Graphical calculation procedures such as the one proposed in this work can be interesting alternatives in response to the needs of engineering contexts with little software support. They could also be helpful when software tools are in use, but the result of a numerical simulation must be quickly verified or there is no time for building the required geometric models.

Apart from this practical value, the proposed method does not imply the proposal of a novel theoretical concept for tolerance analysis. The properties of error buildup that are exploited by the static analogy are likely to correspond to the mathematical properties of existing methods and may thus be hidden in currently available software tools.

Even without a rigorous proof, the analogy between linearised tolerance chains and free-body diagrams of force analysis has been demonstrated to be correct on conceptual and application cases at various difficulty levels. The procedure derived from the analogy appears to be very easy to apply and learn because it is close to other analysis tasks in which mechanical designers are routinely involved. Although there are certain problems to be solved in geometric reasoning, the procedure could also be integrated into software tools for the static analysis of mechanisms and structures, thus allowing for 
the treatment of three-dimensional tolerance chains, which would be difficult to tackle by purely graphical means.

In its current state, the procedure is no more than a quick and dirty solution to a limited class of tolerance chains. The practical application of the procedure in any form is obviously conditioned to the release of the assumptions made in this work. Specifically, possible analogies will have to be investigated for angular dimensions and geometric characteristics, which may be assigned tolerances according to the GD\&T approach; additional analogies might be recognised in other types of entities that are usually considered in statics, such as torque and rotation. Conversely, it will be interesting to understand how the concepts deriving from the statics of deformable bodies could be applied to the analysis of tolerances on overconstrained assemblies.

\section{References}

Ballot, E. and Bourdet, P. (1997), "A computation method for the consequence of geometric errors in mechanisms", Proceedings CIRP International Seminar on Computer-Aided Tolerancing, Toronto.

Bourdet, P. and Ballot, E. (1995), "Geometrical behavior laws for computer-aided tolerancing", Proceedings CIRP International Seminar on Computer-Aided Tolerancing, Tokyo.

Bourdet, P., Mathieu, L., Lartigue, C. and Ballu, A. (1995), "The concept of the small displacement torsor in metrology", Proceedings International Euroconference on Advanced Mathematical Tools in Metrology, Oxford.

Chase, K.W., Gao, J. and Magleby, S.P. (1994), "General 2-D tolerance analysis of mechanical assemblies with small kinematic adjustments", ADCATS Report 94-1, Brigham Young University.

Chase, K.W., Gao, J., Magleby, S.P. and Sorensen, C.D. (1994), "Including geometric feature variation in tolerance analysis of mechanical assemblies", ADCATS Report 94-3, Brigham Young University.

Chase, K.W., Magleby, S.P. and Glancy, C.G. (1997), “A comprehensive system for computer-aided tolerance analysis of 2-D and 3-D mechanical assemblies", Proceedings CIRP International Seminar on ComputerAided Tolerancing, Toronto.

Chiesi, F. and Governi, L. (2003), "Tolerance analysis with eM-TolMate", Journal of Computing and Information Science in Engineering, Vol. 3, pp. 100-105.

Desrochers, A., Ghie, W. and Laperrière, L. (2003), "Application of a unified Jacobian-torsor model for tolerance analysis", Journal of Computing and Information Science in Engineering, Vol. 3 No. 1, pp. 2-14.

Desrochers, A. and Rivière, A. (1997), "A matrix approach to the representation of tolerance zones and clearances", International Journal of Advanced Manufacturing Technology, Vol. 13, pp. 630-636.

Faerber, P.J. (1999), "Tolerance analysis of assemblies using kinematically derived sensitivities", ADCATS Report 99-3, Brigham Young University.

Fischer, B.R. (2004), Mechanical Tolerance Stackup and Analysis, Marcel Dekker, New York.

Gao, J., Chase, K.W. and Magleby, S.P. (1998), "General 3-D tolerance analysis of mechanical assemblies with small kinematic adjustments", IIE Transactions, Vol. 30, pp. 367-377.

Giordano, M. and Duret, D. (1993), "Clearance space and deviation space: application to three-dimensional chain of dimensions and positions", Proceedings CIRP International Seminar on Computer-Aided Tolerancing, Cachan. 
Gupta, S. and Turner, J.U. (1993), "Variational solid modeling for tolerance analysis", IEEE Computer Graphics and Applications, Vol. 13 No. 3, pp. 64-74.

Hunt, K.H. (1978), Kinematic Geometry of Mechanisms, Oxford University Press, New York.

Huo, H. (1995), "New tolerance analysis methods for preliminary design of mechanical assemblies", ADCATS Report 95-4, Brigham Young University.

Lafond, P. and Laperrière, L. (1999), "Jacobian-based modeling of dispersions affecting pre-defined requirements of mechanical assemblies", Proceedings IEEE International Symposium on Assembly and Task Planning, Porto.

Legoff, O., Villeneuve, F. and Bourdet, P. (1999), "Geometrical tolerancing in process planning: a tridimensional approach", Proceedings of the IMechE Part B, Vol. 213, pp. 635-640.

Mantripragada, R. and Whitney, D.E. (1998), "The datum flow chain: a systematic approach to assembly design and modeling", Research in Engineering Design, Vol. 10, pp. 150-165.

Mantripragada, R. and Whitney, D.E. (1999), "Modeling and controlling variation propagation in mechanical assemblies using state transition models", IEEE Transactions on Robotics and Automation, Vol. 15 No. 1, pp. 124-140.

Marziale, M. and Polini, W. (2009), “A review of two models for tolerance analysis of an assembly: vector loop and matrix”, International Journal of Advanced Manufacturing Technology, Vol. 43, pp. 1106-1123.

Marziale, M. and Polini, W. (2010), “A review of two models for tolerance analysis of an assembly: Jacobian and torsor”, International Journal of Computer Integrated Manufacturing, Vol. 24 No. 1, pp. 74-86.

Ohwovoriole, M.S. and Roth, B. (1981), “An extension of screw theory”, ASME Journal of Mechanical Design, Vol. 103, pp. 725-735.

Polini, W. (2009), "Geometric tolerance analysis", in Colosimo, B. and Senin, N. (Eds.), Geometric Tolerances, Springer, London, pp. 39-68.

Prisco, U. and Giorleo, G. (2002), "Overview of current CAT systems", Integrated Computer-Aided Engineering, Vol. 9, pp. 373-387.

Salomons, O.W., Haalboom, F.J., Jonge Poerink H.J., van Slooten, F., van Houten, F.J.A.M. and Kals, H.J.J. (1996), “A computer-aided tolerancing tool II: tolerance analysis", Computers in Industry, Vol. 31, pp. 175-186.

Shah, J.J., Ameta, G., Shen, Z. and Davidson, J. (2007), "Navigating the tolerance analysis maze", ComputerAided Design and Applications, Vol. 4 No. 5, pp. 705-718.

Shen, Z., Ameta, G., Shah, J.J. and Davidson, J.K. (2005), "A comparative study of tolerance analysis methods", Journal of Computing and Information Science in Engineering, Vol. 5, pp. 247-256.

Shen, Z. (2003), "Tolerance analysis with EDS/VisVSA", Journal of Computing and Information Science in Engineering, Vol. 3, pp. 95-99.

Sodhi, R. and Turner, J. (1994), "Relative positioning of variational part models for design analysis", ComputerAided Design, Vol. 26 No. 5, pp. 366-378.

Teissandier, D., Couétard, Y. and Gérard, A. (1999), “A computer aided tolerancing model: proportioned assembly clearance volume”, Computer-Aided Design, Vol. 31, pp. 805-817.

Timoshenko, S. and Young, D.H. (1983), Engineering Mechanics, 4th Edition, McGraw-Hill, New York. 
Figures
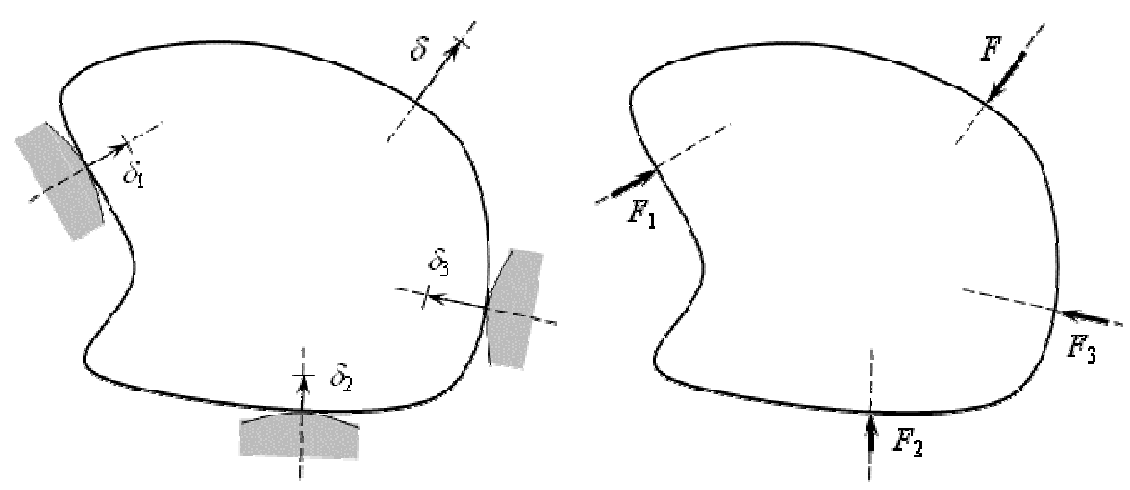

Figure 1 Static analogy of the statkup of dintensimal entors

a)

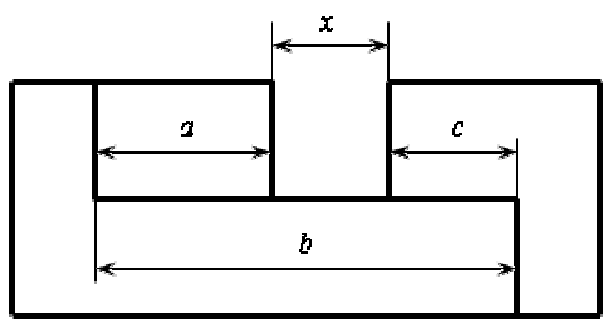

c)

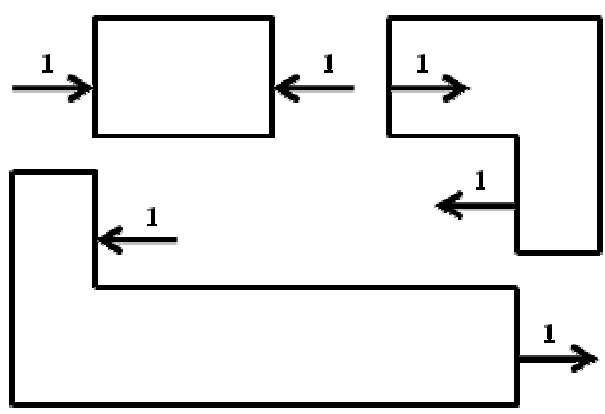

b)
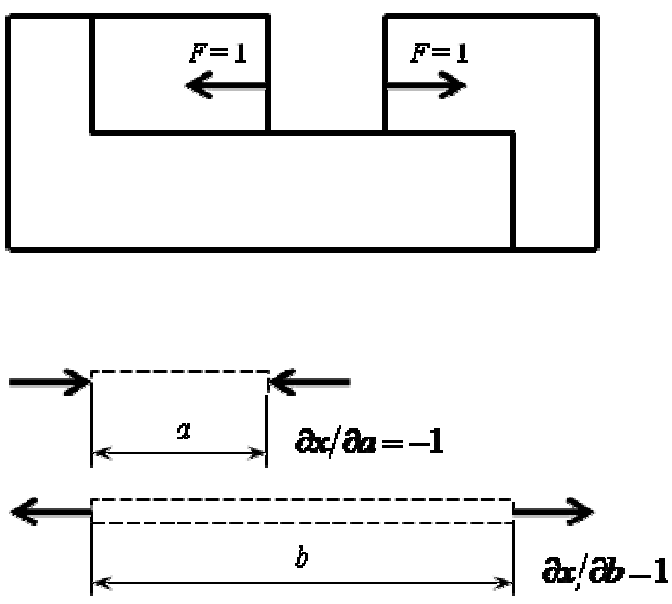

d)

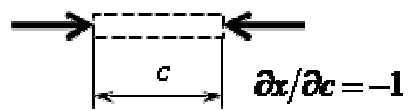

Figure 2 Oulline of the tolerane analy sis provedure 
a)
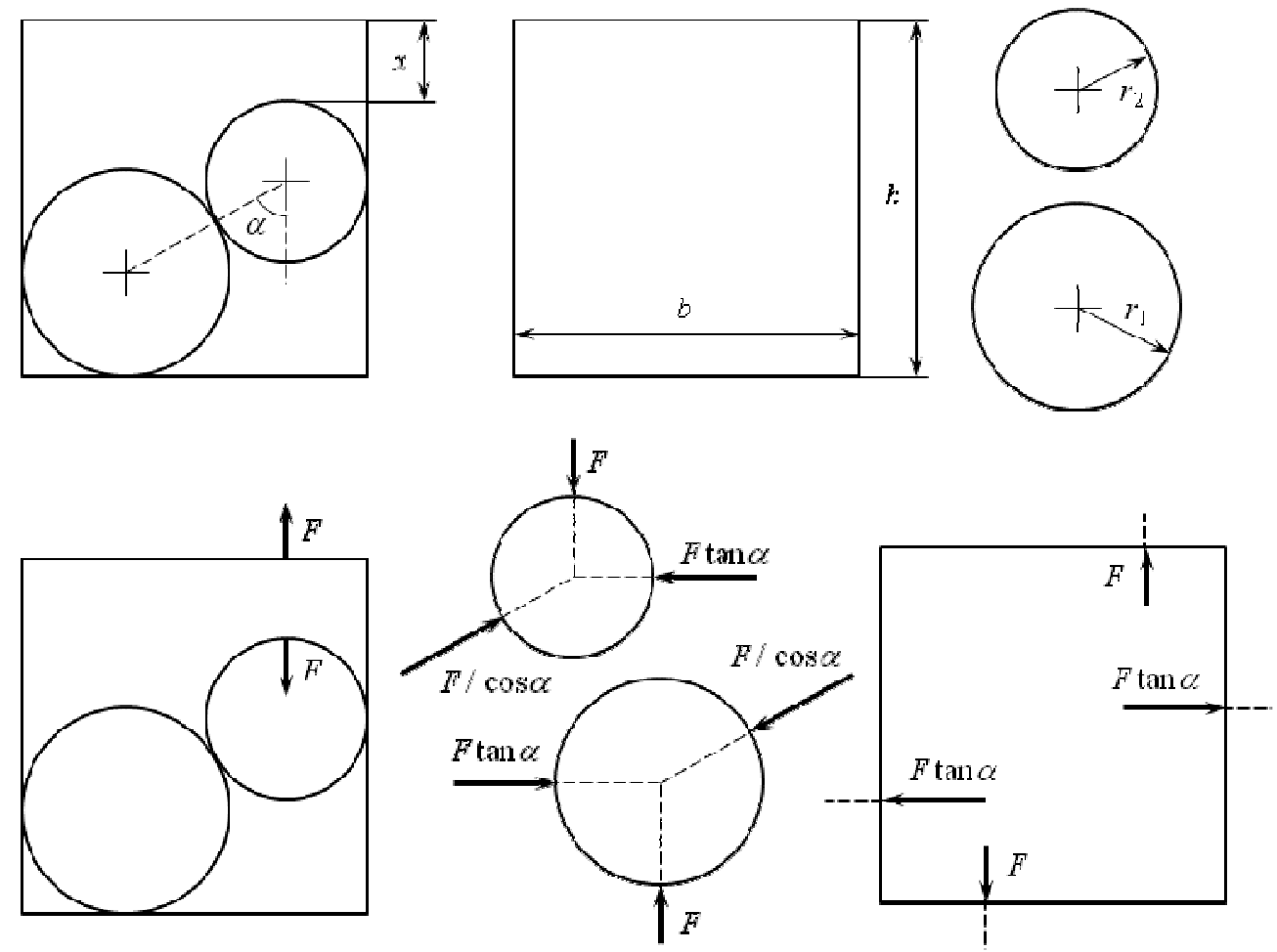

Figure 3 Example for comparison to explicit equation

a)

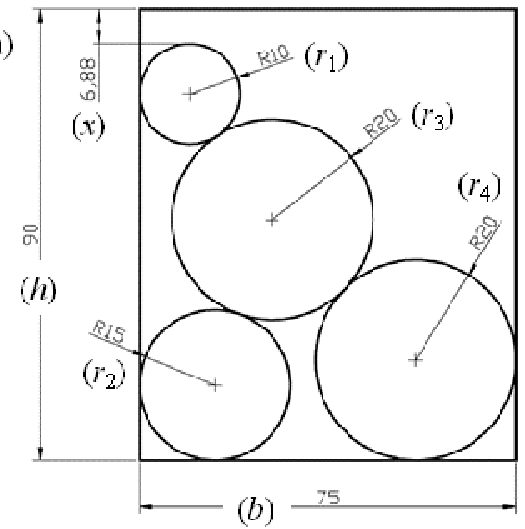

c)
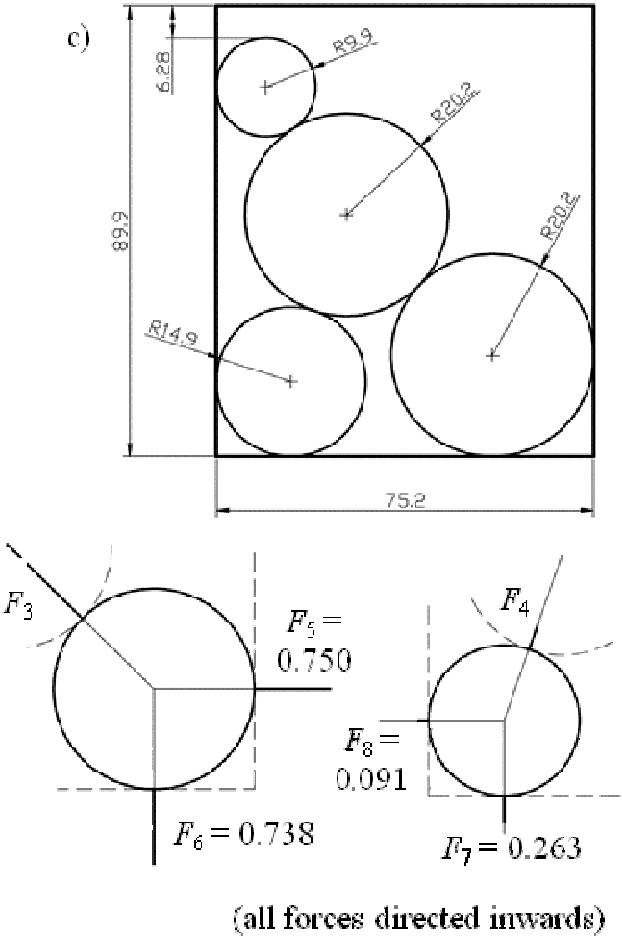

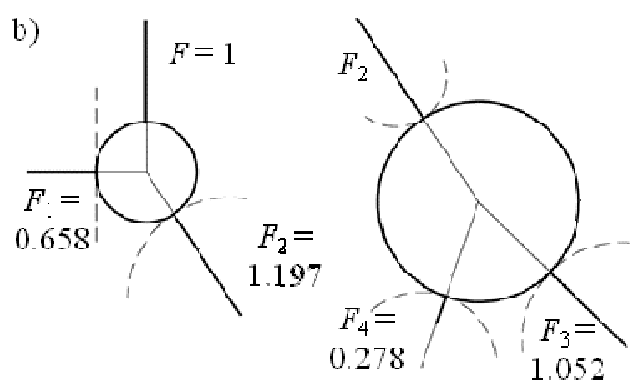

Figure 4 Example for compaison to graphical results 


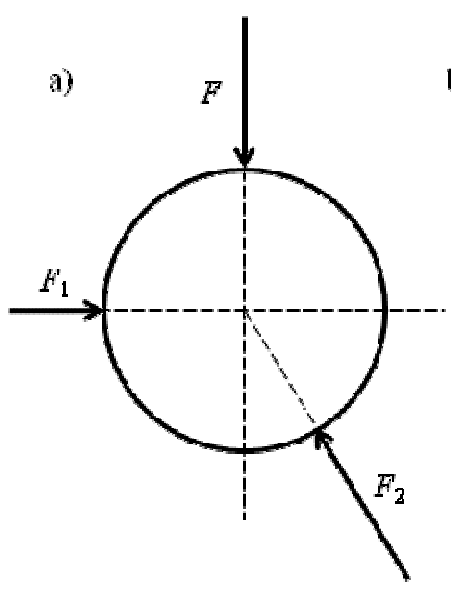

b)
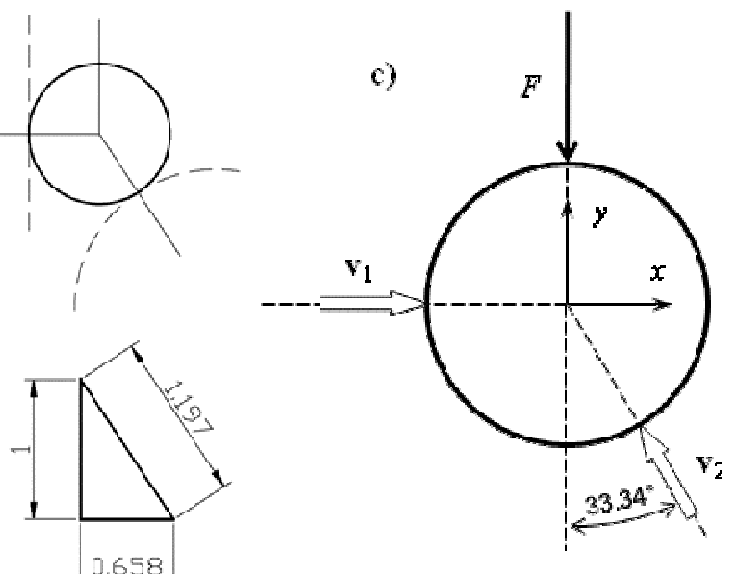

Flgure 5 Graphical and analytical calculations of reaction forces

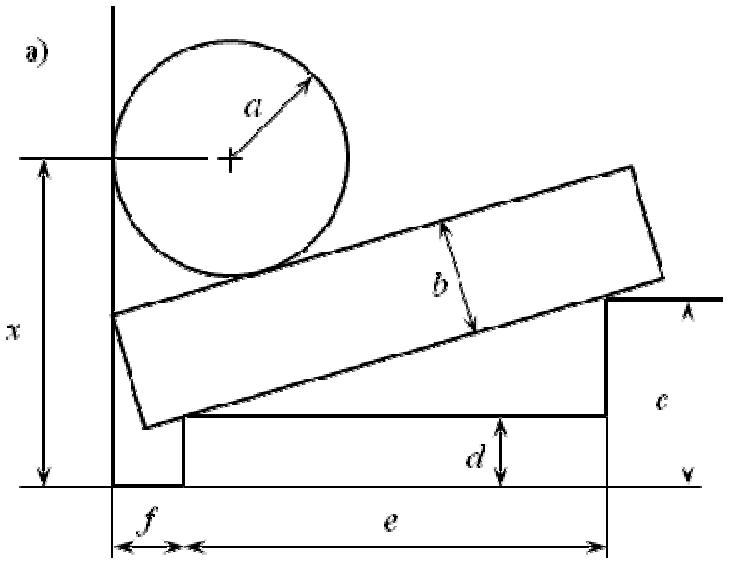

\begin{tabular}{|l|l|}
\hline$a$ & 6.620 \\
\hline$b$ & 6.805 \\
\hline$c$ & 10.675 \\
\hline$d$ & 4.060 \\
\hline$e$ & 24.220 \\
\hline$f$ & 3.905 \\
\hline
\end{tabular}
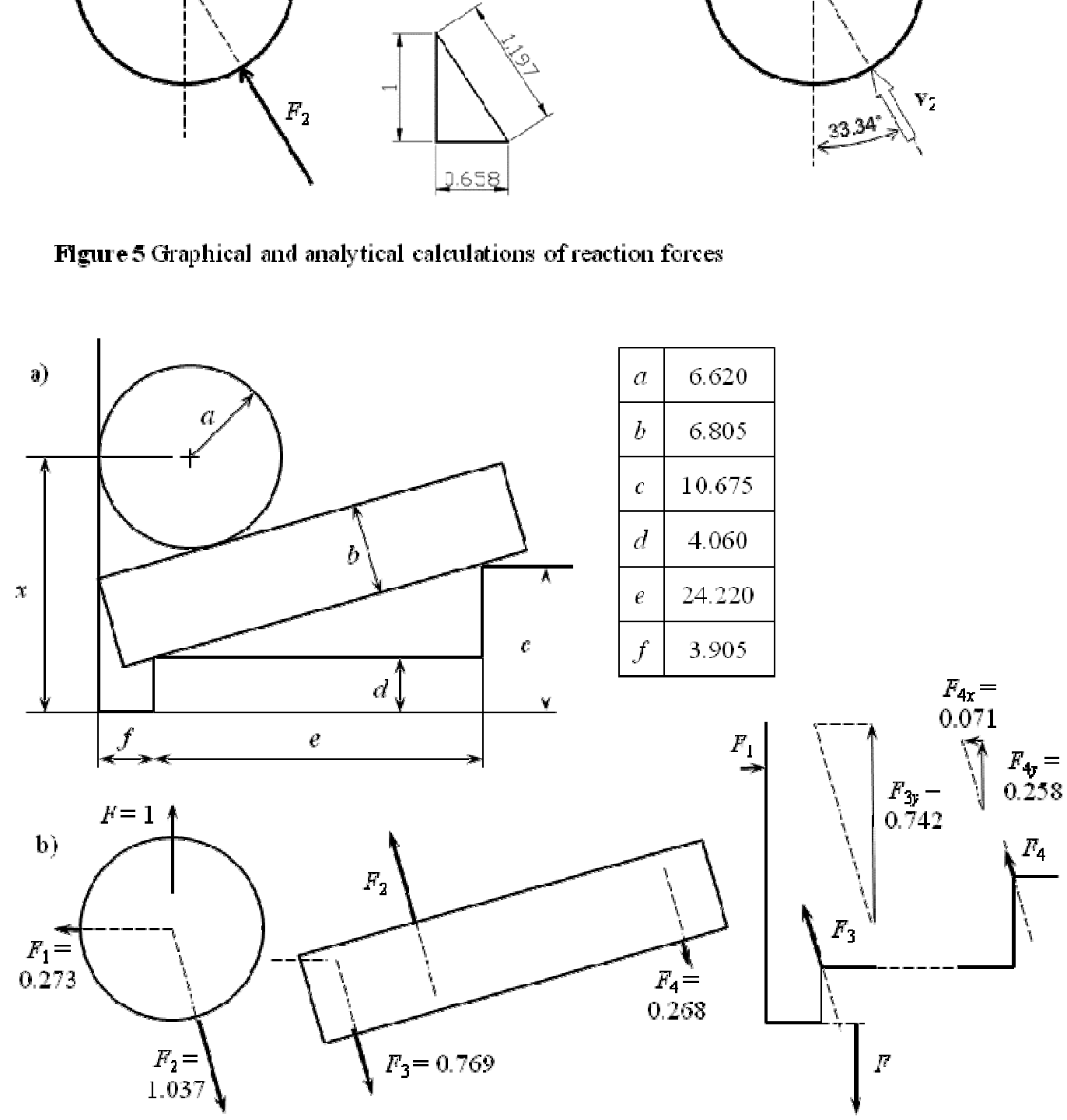

Figure 6 Example for comparison to existing method 

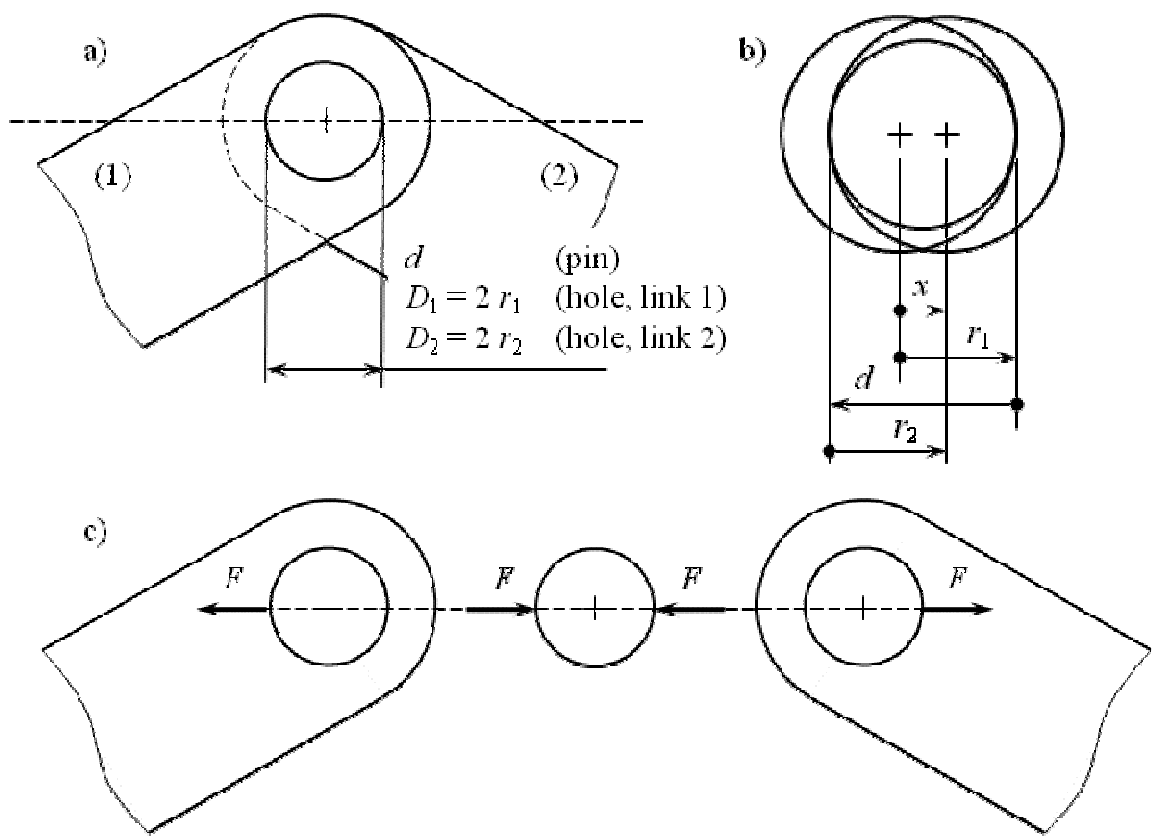

Figure 7 Application of the procedure to hinged mechanisms
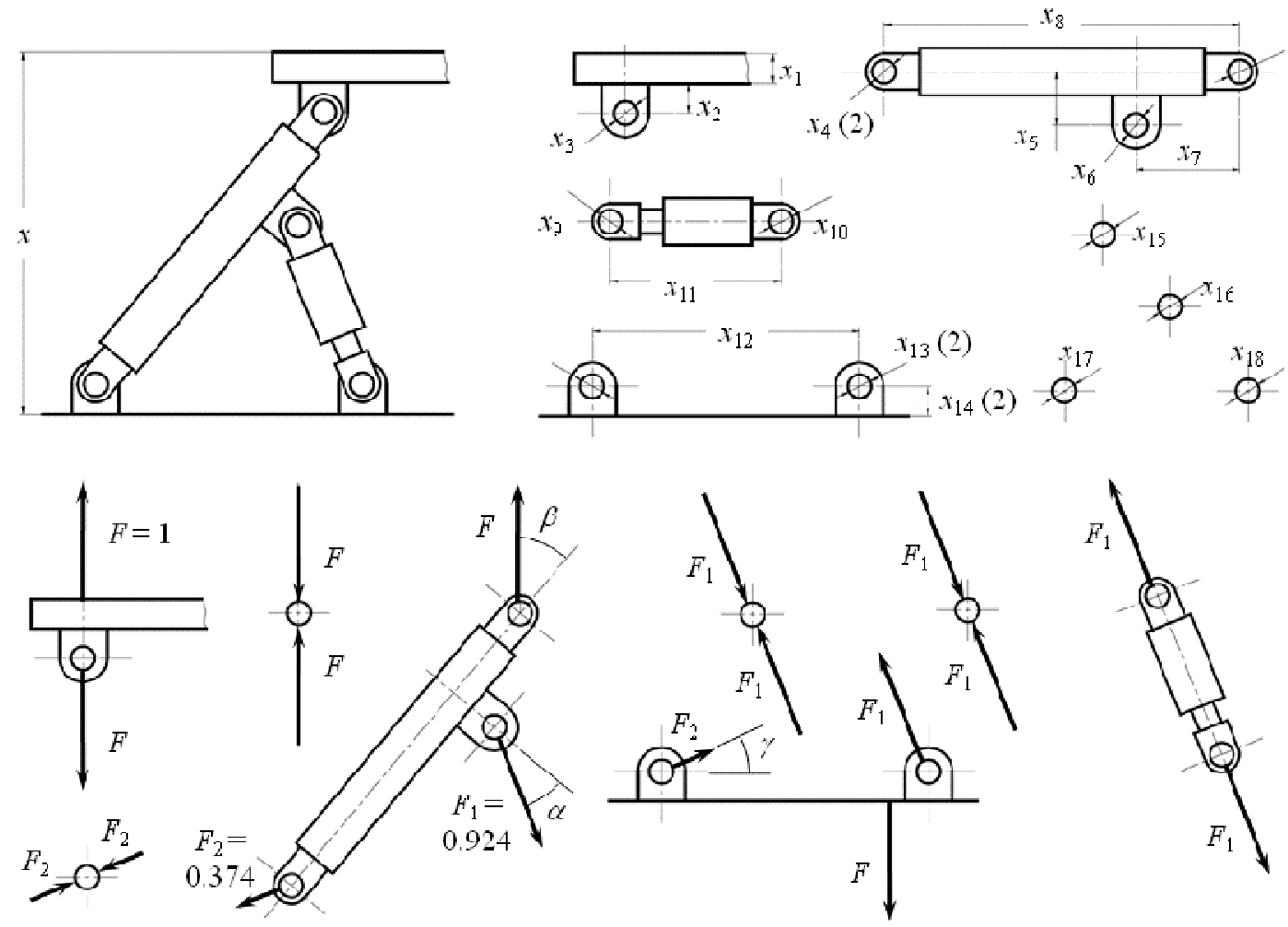

Figure 8 Analy sis of a planar linkage 


\section{Tables}

Tab. 1 Evaluation of sensitivities for the planar linkage example

\begin{tabular}{ccc}
\hline Sensitivities & Expression & Value \\
\hline$s_{1}, s_{2}, s_{14}$ & $F$ & 1 \\
$s_{3}$ & $F / 2$ & 0.5 \\
$s_{4}$ & $F / 2+F_{2} / 2$ & 0.687 \\
$s_{5}$ & $F_{1} \cos \alpha$ & 0.816 \\
$s_{6}, s_{9}, s_{10}$ & $F_{1} / 2$ & 0.462 \\
$s_{7}$ & $F_{1} \sin \alpha$ & 0.434 \\
$s_{8}$ & $F \cos \beta$ & 0.766 \\
$s_{11}$ & $F_{1}$ & 0.924 \\
$s_{12}$ & $-F_{2} \cos \gamma$ & -0.347 \\
$s_{13}$ & $F_{1} / 2+F_{2} / 2$ & 0.649 \\
$s_{15}$ & $-F$ & -1 \\
$s_{16}, s_{18}$ & $-F_{1}$ & -0.924 \\
$s_{17}$ & $-F_{2}$ & -0.374 \\
\hline
\end{tabular}

